\title{
A ATUAÇÃO DO BIBLIOTECARIO MÉDICO E SUA INTERAÇÃO COM OS PROFISSIONAIS DA SAÚDE PARA BUSCA E SELEÇÃO DE INFORMAÇÃO ESPECIALIZADA
}

\section{Fabiano Couto Corrêa da Silva}

" Aquele que sabe onde encontrar a ciência assemelha-se àquele que a possui. "

(A. Mikailov)

\section{Resumo}

Este artigo apresenta e discute as atividades do Bibliotecário Médico, ao mesmo tempo em que descreve os principais recursos informacionais existentes para atender a demanda informacional dos profissionais da área da saúde e a aplicação dos resultados levantados pelo bibliotecário para aplicar o uso efetivo da Medicina Baseada em Evidências.

Palavras-chave

Bibliotecário médico; Medicina baseada em evidências; Bases de dados; Fontes de informação; Profissionais da saúde.

\section{THE PERFORMANCE OF THE MEDICAL LIBRARIAN AND ITS INTERACTION WITH THE HEALTH PROFESSIONALS FOR RESEARCH AND SELECTION OF SPECIALIZED INFORMATION}

\begin{abstract}
This article discusses the activities of Medical Librarian, at the same time that describes the main information resources existing for attending the informational demand from the health professionals and the application of the obtained results by the librarian to aplly the effective use of the Medicine Based in Evidences.

\section{Keywords}

Medical librarian; Medicine based in evidences; Database; Information sources; Health professionals.
\end{abstract}




\section{RELATO DE EXPERIÊNCIA}

\section{INTRODUÇÃO}

Mesmo não trabalhando diretamente com os pacientes, o bibliotecário médico exerce um papel de grande importância na utilização da informação para melhorar a qualidade do atendimento realizado pelos médicos e demais profissionais envolvidos com a assistência à saúde da população.

Pelo fato de não ser um membro da profissão médica, precisa trabalhar em conjunto com todos os tipos de profissionais da área da saúde para garantir o sucesso de suas pesquisas. A sua parceria com outros profissionais qualifica o atendimento aos pacientes e agrega muito valor na obtenção de resultados mais eficazes para os atendimentos clínicos, estudos acadêmicos e desenvolvimento científico.

Os bibliotecários médicos auxiliam os demais profissionais da área da saúde, ajudando-os na busca de casos semelhantes ao problema do paciente, buscando fontes de pesquisa que auxiliam o diagnóstico do médico (terapeutas, farmacêuticos, enfermeiros, veterinários, pesquisadores, etc.), pesquisas acadêmicas dos estudantes de medicina, e leva informação sobre saúde às pessoas através de diferentes canais de comunicação, tais como portais na Internet e o acervo de unidades de informação.

As investigações realizadas em parceria entre o bibliotecário médico e os profissionais da saúde para encontrar informações em literatura especializada podem trazer resultados muito satisfatórios para o atendimento médico. Algumas vezes, as clínicas não possuem conhecimento suficiente para buscar a informação mais adequada ao problema do paciente em publicações científicas, desconhecendo as melhores fontes de pesquisa. Devido à produção crescente de novas pesquisas científicas, pode ocorrer uma desatualização gradual do conhecimento médico utilizado em uma clínica para aplicar diagnósticos e receitas médicas, caso não haja um acompanhamento da evolução científica através de literatura especializada. A maioria dos médicos não adquire as habilidades necessárias em sua formação acadêmica para realizarem buscas de literatura especializada nas melhores fontes de pesquisa. As técnicas utilizadas pelo bibliotecário médico para encontrar em bases de dados, Internet e em literatura especializada os dados científicos de experiências e 
investigações, podem contribuir significativamente nas chances de um diagnóstico preciso sobre o problema de saúde (ou psicológico) apresentado por um paciente. Cabe ao médico avaliar o levantamento bibliográfico realizado pelo bibliotecário e determinar a aplicação dos dados recebidos ao caso de um paciente específico, ou solicitar novas investigações, a fim de obter um aprofundamento ainda maior da pesquisa realizada. Essa parceria entre o bibliotecário e os profissionais da saúde, desenvolve um trabalho que agrega valor para as duas atividades, ocasionando uma troca mútua entre o desenvolvimento das pesquisas realizadas pelo bibliotecário através da análise dos resultados obtidos pelos profissionais da saúde e sua aplicação ao paciente.

\section{A atuação do bibliotecário médico}

No campo da biblioteconomia médica, os Estados Unidos destacam-se pelas iniciativas na formação de bibliotecários médicos: em 1939, a biblioteconomia médica foi reconhecida como uma profissão; em 1947, a Medical Library Association (MLA) adotou um programa de treinamento que foi oferecido na Columbia University School of Library Service em Nova York, com aulas ministradas pelos professores que faziam parte do College of Physicians and Surgeons. O segundo curso foi na Emory University, em Atlanta, em 1951, que também incluía aulas no curso de medicina.

Em 1990, foi criada nos Estados Unidos, a AMIA (American Medical Informatics Association), uma organização sem fins lucrativos, a qual estão associados pessoas, instituições e corporações dedicadas ao desenvolvimento e uso de tecnologias da informação para melhorar o cuidado com a saúde. A AMIA teve a sua origem através da fusão de três organizações - a American Association for Medical Systems and Informatics (AAMSI), o American College of Medical Informatics (ACMI) e o Symposium on Computer Applications in Medical Care (SCAMC). Desde a sua origem, a associação tem contribuído significativamente para o progresso do desenvolvimento de pesquisas na área de saúde, além de funcionar como uma excelente oportunidade para que profissionais de diferentes áreas possam unir-se em torno de um objetivo em comum. Hoje, a AMIA possui 


\section{RELATO DE EXPERIÊNCIA}

3.200 membros, dos quais estão incluídos profissionais médicos, enfermeiros, cientistas da computação e da informação, engenheiros biomédicos, bibliotecários médicos e educadores e pesquisadores acadêmicos.

No Brasil, sem vínculos com a formação acadêmica, os profissionais com atuação em áreas comuns, se reúnem em grupos ou em associações de bibliotecários, a fim de compartilhar experiências e trocar conhecimentos sobre ferramentas de busca da informação especializada na área médica.

A graduação em biblioteconomia proporciona uma formação generalista, e além dos programas de pós-graduação strictu sensu, algumas instituições oferecem cursos voltados especialmente para a área biomédica e de ciências da saúde.

O mais recente deles é primeiro Curso de Especialização em Informações em Ciência da Saúde para Bibliotecários e Documentalistas, de responsabilidade da Universidade Federal de São Paulo (UNIFESP) ${ }^{1}$, no modelo de pós-graduação lato sensu.A Biblioteca Central do Hospital do Servidor Público Estadual participa do programa de aprimoramento da Fundação de Amparo à Pesquisa de São Paulo - FUNDAP e oferece o curso de Aprimoramento Profissional na Área da Saúde, com duração de dois anos, desde 1984 e, a partir de 2000, com duração de um ano.

No Brasil, os bibliotecários médicos desempenham suas atividades nas bibliotecas médicas de instituições de ensino ou de saúde e seus clientes são geralmente, além da comunidade externa, estudantes, professores, pesquisadores, e profissionais da saúde. Bibliotecários médicos são importantes parceiros das equipes de cuidados de saúde, nas pesquisas médicas e na educação dos profissionais de saúde, assim como no fornecimento de informação de alta qualidade para o público em geral.

Segundo a Medical Library Association (MLA), existente nos Estados Unidos, os bibliotecários médicos são responsáveis por muitas tarefas, dentre elas:

\footnotetext{
${ }^{1} \mathrm{http}: / /$ procdados.epm.br/dpd/proex/index.htm 
$\Rightarrow$ Desenhar, desenvolver, navegar e manter sites;

$\Rightarrow$ Localizar recursos impressos e eletrônicos;

$\Rightarrow$ Criar e administrar produtos e serviços que proporcionam informação facilmente;

$\Rightarrow$ Selecionar e comprar livros, revistas e vídeos (em formato impresso e eletrônico);

$\Rightarrow$ Organizar livros, revistas e recursos eletrônicos para o uso rápido e fácil;

$\Rightarrow$ Ensinar os outros a utilizar os computadores, a web e outros sistemas a fim de encontrar a informação que necessitam;

$\Rightarrow$ Realizar empréstimo entre diferentes unidades de informação da área da saúde;

$\Rightarrow$ Servir à sociedade mediante a localização da informação para melhorar o serviço à população.

Os bibliotecários médicos trabalham em bibliotecas ou em centros de informação localizados em:

$\Rightarrow$ Universidades comunitárias e universidades de ensino superior;

$\Rightarrow$ Corporações (por exemplo: em companhias de publicações, de seguros e farmacêuticas);

$\Rightarrow$ Hospitais e clínicas;

$\Rightarrow$ Agências governamentais;

$\Rightarrow$ Portais de Internet;

$\Rightarrow$ Bibliotecas públicas;

$\Rightarrow$ Centros de pesquisa e fundações.

\section{O mercado de trabalho para o bibliotecário médico na Internet}

Através da Internet, o bibliotecário médico pode criar e buscar muitas oportunidades para atuar em sua especialidade. Por meio da obtenção e disponibilização de informações em meio virtual, o profissional bibliotecário pode obter excelentes resultados em suas pesquisas e gerenciar o acesso e conteúdo disponível da unidade de informação em que trabalha, ordenando todo o referencial teórico que pretende disponibilizar. Na página do Conselho Federal de Medicina (www.portalmedico.org.br), existe um link com as regras básicas para que um site tenha uma linha ética de trabalho. Entre os pontos abordados, está a obrigatoriedade de deixar claro para o internauta, na primeira página, quais as áreas para profissionais, pacientes e imprensa. É possível encontrar essa estrutura recomendada pelo Conselho Federal de Medicina no site do Centro de Informações Toxicológicas de Santa Catarina (CIT/SC), onde estão disponíveis todas as áreas sugeridas e, inclusive, a estrutura dos profissionais que compõem o atendimento ao público, como o bibliotecário médico e 
os demais profissionais da área de saúde (médicos, enfermeiros e farmacêuticos). No site do CIT/SC, também é possível encontrar informações sobre toxicologia de interesse do público em geral, e que podem ser muito úteis ao aprendizado sobre os temas apresentados, como envenenamentos e intoxicações.

Os bibliotecários, médicos e pacientes podem se beneficiar em todos os aspectos com a revolução gerada pelo acesso rápido e eficaz decorrente do uso cada vez mais difundido da Internet. Os médicos e profissionais da saúde mais informados (com acesso às novidades da área on-line) têm melhores condições de acertar na sua tomada de decisões acerca do diagnóstico médico e providências a serem seguidas. Atualmente, o acesso a essas informações não é mais monopólio da equipe de saúde, pois a cada dia cresce o número de pacientes que "varrem" a Internet em busca de informações sobre sua saúde e, consequentemente, exigem maior qualidade e clareza no atendimento médico.

A grande vantagem dos portais é reunir em um único espaço diversos assuntos. Esse pode ser o primeiro passo para iniciar a pesquisa. As publicações médicas de revistas de referência demonstram ser ferramentas cada vez mais importantes para os profissionais da área de saúde divulgarem as suas pesquisas, tornando os resultados de seus estudos acessíveis mais rapidamente para todos os pesquisadores, como o bibliotecário médico, que precisa estar atento às novidades para repassar aos seus usuários. O Salutia (www.salutia.com), por exemplo, é um portal de saúde latino-americano (em português e espanhol) com uma grande variedade de conteúdo, produzido por médicos e jornalistas. No Salutia, para saber sobre alguma doença, há duas formas de pesquisa: através do sistema de buscas - é mais rápido e trará vários conteúdos e artigos sobre o assunto. Outra forma, é acessar o link Doença, item Tudo Sobre, que encontra-se no canto inferior esquerdo da tela. O site Saúde Vida On-line (www.saudevidaonline.com.br) é uma revista eletrônica com uma proposta bem interessante: as seções estão divididas em saúde da mulher, do homem, filhos, adolescentes, terceira idade e olhos. Há muitos artigos escritos por médicos, que servem como guia das doenças mais comuns. 
O serviço E Agora Doutor? se destaca pela variedade de temas. Há desde medicina convencional até chinesa, esportiva, do trabalho, fonoaudiologia, psicologia e outras. Apesar disso, não há um mecanismo de buscas para facilitar as pesquisas.

Até o Doutor Drauzio Varella, conhecido por seus livros e programas em rádio e tevê, tem um site (www.drauziovarella.com.br). Na página, há artigos sobre alcoolismo, asma/bronquite, enfarte e enfisema pulmonar, além de entrevistas. Esta, aliás, é a melhor seção do site, mas, por enquanto, traz apenas três temas: problemas do sono, aleitamento materno e calvície.

Porém, sabe-se que existem sites de saúde que baseiam o seu conteúdo em dados nãocientíficos, fazendo com que leigos no assunto tenham acesso a informações desatualizadas e muitas vezes erradas, já que não existe fiscalização ou controle sobre o que é lançado na rede. Alguns sites oferecem até diagnóstico clínico por meio de correio eletrônico. Portanto, o bibliotecário médico precisa saber pesquisar os dados desejados em sites que possuem informações seguras e corretas sobre doenças, medicações e qualquer outro assunto que trate de saúde, recomendando sempre ao leigo buscar referências com um profissional de saúde de confiança. Cabe lembrar, que uma "consulta virtual" não pode substituir a visita ao médico, pois somente ele é quem tem autoridade de, após exame clínico, diagnosticar alguma patologia e promover o seu tratamento.

Dessa forma, o desenvolvimento do trabalho do bibliotecário médico através da Internet e pela busca no acervo impresso, somente será possível através de uma revisão técnica por um profissional ou equipe de profissionais de saúde especializados, os quais terão conhecimentos para avaliar o conteúdo informacional pesquisado pelo bibliotecário médico. Essa avaliação dar-se-á através das técnicas de busca utilizadas pelo bibliotecário, e a qualidade dos resultados dependerá da troca mútua entre as duas atividades profissionais. 


\section{RELATO DE EXPERIÊNCIA}

\section{Medicina Baseada em Evidências (MBE)}

A Medicina Baseada em Evidências (MBE) foi formulada e desenvolvida por um grupo de pesquisadores da Universidade MacMaster, do Canadá, no início da década de 80. Consiste em uma metodologia de localização, avaliação e uso de descobertas recentes em medicina como base para as atividades cotidianas de um médico. Sua prática pode agregar muito valor no atendimento médico, e até mesmo tornar-se o motivo do sucesso de análises clínicas difíceis de serem diagnosticadas.

Os seus quatro passos principais são: formular uma questão objetiva; procurar artigos relevantes na literatura; avaliar sua validade e utilidade; implementar os achados na prática clínica. A MBE não é uma especialidade ou ramo da medicina, mas um procedimento que pode ser utilizado pelo bibliotecário, em qualquer área a que ele se dedique, servindo como instrumento ao médico sempre que houver uma dúvida no diagnóstico, no prognóstico ou no tratamento de doenças.

A MBE se apóia em 3 áreas básicas: epidemiologia clínica, bioestatística e informática médica. A epidemiologia clínica fornece os instrumentos para a elaboração e análise de experimentos científicos que permitam obter evidências sólidas sobre questões específicas formuladas. A bioestatística, com seu conjunto de métodos para avaliação dos dados quantitativos da pesquisa científica, permite dimensionar e categorizar a força das evidências encontradas. Por fim, a informática médica permite, hoje em dia, a busca de forma extensiva na literatura médico-científica dos trabalhos publicados que apresentem as melhores evidências, base para as decisões de cunho terapêutico ou preventivo.

A Medicina Baseada em Evidências (MBE) é um movimento médico que se baseia na aplicação do método científico a toda prática médica, especialmente às tradições médicas tradicionalmente estabelecidas que ainda não foram submetidas ao escrutínio sistemático científico. Evidências significam, aqui, provas científicas.

Como processo, a MBE se inicia pela formulação clara do problema, que deve ser o mais específico possível, a pesquisa de forma sistemática na literatura médica correspondente, a 


\section{RELATO DE EXPERIÊNCIA}

avaliação crítica das evidências obtidas, tanto no que diz respeito à sua força, quanto à sua aplicabilidade e, por fim, a aplicação das evidências encontradas, seja na forma de intervenções práticas ou na formulação de novos estudos científicos.

Um dos criadores deste movimento foi o professor Archie Cochrane, pesquisador britânico autor do livro Effectiveness and Efficiency: Random Reflections on Health Services (1972). Sua luta levou à crescente aceitação popular do conceito da Medicina baseada em evidências. Seu trabalho foi reconhecido e homenageado com a criação dos centros de pesquisa de medicina baseada em evidências (os Cochrane Centres) e de uma organização internacional chamada de Cochrane Collaboration.

A Cochrane Collaboration tornou-se a primeira base de dados sobre resumos sistematizados de evidências sobre intervenções na área da saúde (http://hiru.mcmaster.ca/COCHRANE/; www.cochrane.org). O Cochrane Database of Systematic Reviews constitui uma base de dados de texto completo e consiste em revisões sistemáticas regularmente atualizadas desde 1991. As revisões são apresentadas em dois tipos: a) revisões completas, atualizadas regularmente; b) protocolos de revisões que estão sendo preparadas. Os artigos são selecionados a partir de estratégias de indexação e busca avançada. Existem grupos colaboradores de revisão e Centros Cochrane em alguns países, além do núcleo inglês. O centro brasileiro funciona na Unidade de Ensaios Clínicos e Metanálise da Escola Paulista de Medicina, Universidade Federal de São Paulo (www.centrocochranedobrasil.org) e conta com a colaboração de pesquisadores, pós-graduandos e interessados em quase todo o país, dando suporte e assistência na realização de revisões Cochrane e contribuindo para a colaboração em vários aspectos. A área do conhecimento neurológico, por exemplo, dispõe de alguns grupos colaboradores de revisão (sobre demência e cognição, epilepsia, esclerose múltipla, esclerose neuromuscular, doença cerebrovascular e distúrbios do movimento). Mais recentemente, o York Centre for Reviews and Dissemination, da Universidade de York, uniu-se à Cochrane Collaboration para formar a Cochrane Library (www.updatesoftware.com/cochrane/cochrane-frame.html) ${ }^{2}$.

${ }^{2}$ Um acordo entre o Centro Cochrane Brasil, Bireme e Organização Pan-Americana da Saúde (PAHO), está disponibilizando a Cochrane Library completa via Internet para os médicos brasileiros, hospitais públicos, 


\section{RELATO DE EXPERIÊNCIA}

A prática da MBE implica não somente conhecimento e experiência clínica, mas também conhecimentos técnicos para procurar, encontrar, interpretar e aplicar os resultados de estudos científicos epidemiológicos aos problemas individuais de seus pacientes. Implica também conhecer como calcular e comunicar os riscos e os benefícios dos diferentes cursos de ação aos seus pacientes.

A MBE torna possível que o médico adquira as informações técnicas indispensáveis sobre novos medicamentos, estatísticas, procedimentos e condutas, mas preserva seu tempo para que ele possa tratar e conversar com o seu paciente. Por exemplo, se um médico oftalmologista diz que não há necessidade de se realizar a assepsia de um aparelho que entra em contato com a córnea de seu paciente antes de usá-lo em outro paciente, ele deverá fornecer uma evidência - uma prova científica - dessa afirmação. Assim, deverá procurar um estudo epidemiológico em que foram acompanhados um número suficiente de pacientes que tiveram o exame realizado após assepsia do aparelho comparado com um número semelhante de outros pacientes que tiveram o exame realizado sem a referida assepsia. Após um certo período de acompanhamento, deverão ser analisados todos os pacientes em busca de alterações de saúde.

Assim, o conceito de que a Medicina deve ser exercida baseada em evidências não se discute, porém, é importante discutir o que pode ser considerado como "evidência". Por exemplo, quando o balconista de uma farmácia prescreve antibióticos para o tratamento de um quadro gripal, de acordo com ele esta prescrição está baseada na "evidência" de que todos indivíduos que ele tratou dessa maneira curaram-se e, portanto, este é um tipo de Medicina baseado em evidências. Outro exemplo muito comum é aquele médico que diz que na sua experiência pessoal a conduta $\mathrm{X}$ é a mais indicada para a patologia $\mathrm{Y}$.

associações médicas, universidades e associações leigas da área de saúde, gratuitamente, desde fevereiro de 2001. Maiores informações podem ser oferecidas diretamente no Centro Cochrane do Brasil www.centro cochranedobrasil.org e da Bireme (PAHO) (www.bireme.br).

Para obter a assinatura em CD-ROM, deve-se entrar em contato com o endereço: Update Software Ltd, Summertown Pavilion, Middle Way, Oxford OX2 7LG, UK; tel +44 1865 513902; fax +44 1865 516918; e-mail: info@cochrane.co.uk; www.update-software.com/cochrane ou Update Software Inc, Suite 21, 1070 South Santa Fe Avenue Vista, CA 92084, USA, tel: +1 760631 5844, e-mail: info@updateusa.com.

(C) Revista Digital de Biblioteconomia e Ciência da Informação,Campinas, v. 3, n. 1, p. 131-151, jul./dez. 2005 - ISSN: 1678-765X. 
Pelo exposto, fica claro que é importante saber analisar que tipo de evidência deve ser levado em conta. A maior parte, senão todas as evidências que devem ser consideradas originam-se em pesquisas científicas, cujos resultados são veiculados por artigos publicados em periódicos especializados.

Na prática clínica quatro situações básicas sempre se repetem: a) diagnóstico; b) tratamento; c) prognóstico; d) prevenção. Cada uma dessas situações compreende cinco componentes específicos: a) acesso à informação, b) avaliação crítica (ou melhor, da qualidade) da literatura, c) principais desenhos de pesquisa clínica, d) métodos estatísticos, e) planejamento de pesquisas clínicas. Em cada uma dessas situações básicas é necessário saber acessar a informação, avaliar a literatura, entender as limitações (vantagens e desvantagens) de cada desenho de estudo, os métodos estatísticos envolvidos com a situação clínica e como planejar pesquisas para que seus resultados sejam válidos.

No uso da MBE, três tipos de pessoas estão envolvidas: o médico, o doente, e o bibliotecário; cada um se diferenciará dos outros pelos conhecimentos e habilidades que possuem em cada um desses componentes; diferenciados pelo "saber como é realizado" e pelo "saber realizar".

Médico: cabe ao médico ou profissional da área da saúde saber como é realizado cada um dos itens básicos (acesso à informação, avaliação da literatura, principais tipos de desenhos de pesquisa, métodos estatísticos, planejamento de pesquisas) e as diretrizes clínicas para que ele possa confiar nas informações produzidas por cada uma das partes. A decisão clínica é o item que este profissional necessita saber fazer. O médico deve ter consciência dos itens envolvidos no processo de tomada de decisão clínica, como eles interagem e como individualizar sua conduta para o doente que está na sua frente.

Doente: cabe ao doente saber como são realizados todos os itens descritos, mas não precisa saber realizar nenhum. Se o doente entende como as informações são geradas pelas 


\section{RELATO DE EXPERIÊNCIA}

pesquisas clínicas, como são interpretadas e aplicadas, será participativo e ativo no processo de tomada de decisão que irá afetá-lo.

Bibliotecário: o bibliotecário, diferente das outras pessoas envolvidas, já está designado de acordo com sua função e conhecimentos, sabe realizar cada um dos itens descritos. Por exemplo, para o método estatístico existe o bioestatístico, para o acesso à informação o bibliotecário.

\section{A decisão clínica (Medicina além das evidências)}

O processo de decisão clínica que é coordenado pelo médico tem que obrigatoriamente levar em consideração três componentes: a) as evidências, dizendo de outra forma, os resultados de pesquisas clínicas de boa qualidade, b) as circunstâncias do atendimento, c) os desejos do doente. Os dois últimos componentes mais a coordenação no processo de tomada de decisão é que é a medicina além das evidências. Não levar em consideração os três componentes é um desvio do comportamento médico, pois poderemos ter uma maior probabilidade de malefício que benefício, além de quebrar com a relação médico-paciente que é um elemento essencial na prática clínica.

Algumas entidades como a ACC (American College of Cardiology), fazem uma triagem de artigos significativos para os médicos, além de publicar alguns "guidelines" que destacam as condutas mais indicadas para diversas patologias. Esses "guidelines" geralmente são disponibilizados na Internet. Além disso, são centenas de "papers", "trials", "researches" e "joints" lançados diariamente na rede, acessíveis de casa, do consultório ou do hospital a um custo quase zero. Seria impossível acessar tantos dados sem o auxílio da Internet.

Diante da abrangência da Internet, torna-se um desafio para o bibliotecário discernir qual o material que realmente interessa e que seja confiável, das infomações inúteis. A profusão de dados disponíveis dificultou a seleção e a utilização das informações no dia-a-dia dos médicos. Mas é aí que entra a MBE, como um procedimento para auxiliar os médicos na detecção e utilização daquelas informações que são realmente importantes. Fora da Internet

(C) Revista Digital de Biblioteconomia e Ciência da Informação,Campinas, v. 3, n. 1, p. 131-151, jul./dez. 2005-ISSN: 1678-765X 


\section{RELATO DE EXPERIÊNCIA}

também podemos obter e selecionar essas informações. Alguns livros, como, por exemplo, o Current Medical Diagnosis and Treatment, que é revisado anualmente, também utilizam o conceito de MBE e representam importantes instrumentos para os médicos realizarem suas práticas médicas atualizadas com confiabilidade. O interessante é que esse livro, no final de cada capítulo, também apresenta referências de sites selecionados sobre cada tema. Ou seja, nos remete novamente à rede para uma orientação cada vez mais atualizada.

\section{Revisões Sistemáticas}

As razões para realização das revisões sistemáticas da literatura são:

a) Sintetizar as informações sobre determinado tópico;

b) Integrar informações de forma crítica para auxiliar as decisões;

c) Ser um método científico reprodutível;

d) Determinar a generalização dos achados científicos;

e) Permitir avaliar as diferenças entre os estudos sobre o mesmo tópico;

f) Explicar as diferenças e contradições encontradas entre os estudos individuais;

g) Aumentar o poder estatístico para detectar possíveis diferenças entre os grupos com tratamentos diferentes;

h) Aumentar a precisão da estimativa dos dados, reduzindo o intervalo de confiança;

i) Refletir melhor a realidade.

As revisões sistemáticas têm a vantagem de seguir métodos científicos rigorosos; poderem ser reproduzidas, criticadas, e a crítica incorporada em sua publicação eletrônica. É importante ressaltar, ainda, que com elas se evitam duplicações de esforços, já que, quando feitas uma vez, poderão ser divulgadas e utilizadas mundialmente. Além disso, pode tornarse uma publicação viva, facilmente atualizada de tempos em tempos.

Uma revisão sistemática ${ }^{3}$ deve incluir a procura metódica dos ensaios clínicos existentes (publicados ou não) e o somatório estatístico dos resultados de cada estudo. Esse somatório

\footnotetext{
${ }^{3}$ O centro Cochrane do Brasil em parceria com o Laboratório de Ensino a Distância (LED-Dis) do Departamento de Informática em Saúde da UNIFESP, oferece o Curso de Revisão Sistemática e Metanálise gratuitamente pela Internet (não existe processo seletivo). O curso tem o objetivo de informar as técnicas necessárias para a realização de uma Revisão Sistemática ou Metanálise de intervenções em cuidados da saúde, além de orientar sobre as habilidades necessárias para planejar, conduzir e divulgar a revisão sistemática. Os participantes são orientados durante o processo de planejamento e condução de uma revisão sistemática e terão oportunidade de fazer adaptações para a sua própria revisão. Maiores informaçõs podem ser obtidas diretamente no site www.virtual.epm.br/cursos/metanalise.
}

(C) Revista Digital de Biblioteconomia e Ciência da Informação,Campinas, v. 3, n. 1, p. 131-151, jul./dez. 2005-ISSN: 1678-765X. 
chama-se metanálise, que são estudos em que os pesquisadores juntam pesquisas mundialmente feitas dentro de um período sobre um determinado assunto. Depois eles classificam e aproveitam apenas aquelas cujos experimentos tiveram um bom número de participantes nos testes, com um grupo experimental e um grupo de controle, ou seja, um grupo de participantes que passa pelo procedimento e outro que não passa (o pesquisador não pode saber quem faz parte do grupo de experimentos e quem faz parte do grupo de controle). Por fim, são unidos todos os resultados e é feita a análise estatística desse conjunto de pesquisas. Nesse caso teremos milhares de casos computados e com a certeza de que as pesquisas participantes foram bem planejadas.

Às vezes, autores somam os resultados de alguns artigos, sem a realização da busca metódica de todos os estudos existentes (revisão sistemática), gerando dados discutíveis e causando confusão. A metanálise sem uma revisão sistemática não faz sentido. E condenar a metanálise é equivalente a desacreditar a operação de somar, pelos erros de quem não domina a aritimética elementar. Em outras palavras, as informações devem advir de uma revisão sistemática que deve incluir ou não a metanálise. Da mesma forma que na solução de qualquer problema matemático as operações elementares devem seguir uma lógica aceitável. É por isso, que a Colaboração Cochrane insiste na metodização científica das revisões sistemáticas que são aprimoradas dioturnamente e que por isso são aceitas universalmente como uma das melhores formas de equacionamento de evidências para a pesquisa e para a prática médica. De tal forma que recentemente o Congresso Internacional de Editores de Revistas Médicas, em Praga, considerou o sistema de revisão de pares da Cochrane Library como modelo a ser seguido, fato já posto em prática pelo Lancet, que já avalia e publica os protocolos dos estudos a serem submetidos a ele futuramente (www.thelancet.com).

\section{Guidelines}

Nada mais são que sugestões de condutas clínicas, baseadas nas melhores evidências científicas existentes, produzidas de maneira estruturada. Na ausência de evidências com a qualidade desejada (bons ensaios clínicos, por exemplo) toma-se por base o consenso de 
especialistas no assunto. De forma que, informações relevantes, adequadas para cada situação, são cotadas em relação ao custo-benefício (eficiência) e passam a ser o elo final entre a ciência de boa qualidade e a boa prática médica. Isto tem se tornado rotina nos países desenvolvidos e são dramaticamente necessários nos países em desenvolvimento. É por isso que a Organização Mundial de Saúde criou a WHO Reproductive Human Library, que visa minorar a grave situação materno-infantil nos países em desenvolvimento, onde ocorrem 99\% das mortes maternas do planeta. Nos países desenvolvidos, pode-se exemplificar com o Canadá, Holanda, Reino Unido, Suécia, Estados Unidos e a Austrália, entre outros.

\section{Habilidades}

Uma série de habilidades são necessárias para melhor aproveitamento da Medicina Baseada em Evidências:

a) Definição precisa da questão clínica pelo médico e quais as informações necessárias para respondê-la;

b) Condução de uma busca eficiente da literatura;

c) Seleção dos estudos relevantes e metodologicamente adequados;

d) Apresentação de um resumo estruturado com o conteúdo do artigo.

\section{Fontes de informações baseadas em evidências}

Realizar pesquisas médicas em livros tornou-se uma atitude de enorme risco em decorrência da falta de atualização desse recurso, impossibilitando ao pesquisador encontrar informações clínicas atualizadas e condizentes às necessidades do seu uso pelos profissionais da área de saúde. Na era tecnológica que vivemos atualmente, o conhecimento médico é rapidamente disseminado através da Internet, tornando as pesquisas em livros ultrapassadas, na medida em que o acesso e o uso difundido de informações médicas pela web tornam-se cada vez mais acessíveis e amplos, em diversas áreas do conhecimento. Para que um livro com conteúdo científico seja útil ao bibliotecário como uma ferramenta de pesquisa que possa fornecer um impacto positivo no que diz respeito à sua atualização para 


\section{RELATO DE EXPERIÊNCIA}

os profissionais da saúde, é necessário que ele seja revisado freqüentemente e que tenha muitas referências, possibilitando aos leitores pesquisarem as fontes originais para o aprofundamento de suas pesquisas. O livro Clinical Evidence ${ }^{4}$ oferece esse formato em sua edição, tornando o seu uso bastante atualizado para as pesquisas referentes à prática clínica. Atualmente, este livro inclui apenas evidëncias para tratamento de doenças clínicas. Existem outros textos semelhantes, incluindo um livro, CD e site chamados EvidenceBased on Call que estão sendo desenvolvidos por um consórcio mundial, liderado por um grupo de recém-graduados da universidade de Oxford. Maiores informações podem ser obtidas diretamente no site: (cebm.jr2.ox.ac.uk/eboc.html).

Existem outros livros didáticos com base em evidências. No campo da medicina geral, o $U p$ To Date, atualmente apenas em CD, é atualizado trimestralmente, contém extensas referências e fornece resumos da MEDLINE para a evidência-chave. Isso dá ao usuário pelo menos a chance de datar e analisar a evidência de apoio. O Scientific American Medicine também tem extensas referências e atualmente está sendo ampliado por uma versão na Internet, com conexão às citações e resumos da MEDLINE, assim como outros recursos da Web. O livro-texto Harrinson também está sendo melhorado e fornece mais referências e resumos na sua versão na Web, embora a extensão das referências ainda seja limitada. Essa tendência de livros didáticos mais ricos em citações e com conexões com a Web é encorajadora, mas nenhum desses textos fornece muito no sentido da evidência com análise sistemática e crítica, deixando que os leitores o façam por si mesmos ou esperem que os autores o façam. Observa-se também a emergência de textos mais especializados em evidências, como o Evidence-Based Cardiology, publicado pelo BMJ Publishing Group.

Embora não estejam integradas em torno da área de problemas clínicos de modo conveniente como nos livros didáticos, as melhores evidências atuais de estudos específicos de problemas clínicos podem ser encontradas em número crescente de bancos de dados eletrônicos, alguns com processamento explícito de evidências e outros que deixam o processamento por conta do usuário. Em buscas que exijam mais rigor, recomenda-se o

\footnotetext{
${ }^{4}$ Publicado pela primeira vez em 1999 pela BMJ Publishing Group (www.bmjpg.com/index.html) e pelo American College of Physicians (www.acpon-line.org).
} 


\section{RELATO DE EXPERIÊNCIA}

PubMed (www.ncbi.nlm.hih.gov/PubMed). Sua interface, todavia, não é tão simples quanto parece. Representa uma importante ferramenta em termos de opções de busca e recuperação de dados, mas exige um conhecimento mínimo de quem a utiliza. Para realizar pesquisa no PubMed, há três formas de acesso: a) pesquisa básica; b) pesquisa avançada; c) pesquisa clínica. A pesquisa clínica possibilita que seja utilizado automaticamente uma estratégia de pesquisa previamente montada para cada uma das categorias: terapia, diagnóstico, etiologia, prognóstico. Estas pesquisas pré-programadas têm o objetivo de facilitar a identificação de artigos que ofereçam um bom nível de evidência. Será apenas necessário digitar o termo de pesquisa, escolher a categoria e definir se o resultado da pesquisa será mais sensível ou mais específico. A estratégia pré-programada pode ser observada através do item TABLE. O uso de limites (Limits) oferece acesso fácil aos recursos aqui descritos para tornar a busca mais eficiente e o MeSH Browser permite acessar o vocabulário controlado, suas definições, grafia, etc. Vários outros recursos estão disponíveis, entre eles: Cubby oferece a opção de armazenar as pesquisas realizadas para futuro uso, History permite checar e neutralizar as pesquisas feitas no acesso atual, e o Clipboard permite armazenar referências no acesso atual.

O Evidence-Based Medicine Reviews (EBMR) da Ovid Technologies (www.ovid.com) é um excelente banco de dados. O EBMR combina vários bancos de dados eletrônicos, incluindo bancos de dados especializados como o Cochrane Database of Systematic Reviews, Best Evidence, Evidence-Based Mental Health e Evidence-Based Nursing, Cancerlit, Healthstar, Aidsline, Bioethicsline e MEDLINE, mais conexões com o texto completo de mais de 200 revistas.

O MEDLINE.EBMR interliga esses bancos de dados. Assim, se encontrarmos no MEDLINE um ensaio randomizado sobre um tópico de interesse e esse ensaio foi incluído em uma revisão sistemática na Cochrane Library, será fácil encontrá-lo. O EBMR é particularmente valioso por causa das conexões entre um banco de dados geral (MEDLINE) e os serviços com base em evidências que são preparadas de acordo com princípios e procedimentos explícitos para coleta de evidências conforme a qualidade e o 


\section{RELATO DE EXPERIÊNCIA}

conteúdo. Este serviço é utilizado por muitas bibliotecas das ciências de saúde, sendo muito difundido como ferramenta de trabalho de bibliotecários que atuam na área de saúde.

$\begin{array}{ccccc}\text { A } & \text { Biblioteca } & \text { Cochrane } & \text { (update.cochrane.co.uk; } & \text { www.update-software.com) } \\ \text { e } & \text { a } & \text { Best } & \text { Evidence } & \text { ( } \text { www.update-software.com; }^{2}\end{array}$
www.acponline.org/catalog/eletronic/best_evidence.htm) também estão disponíveis como bancos de dados separados em CD e na Internet. A Biblioteca Cochrane oferece revisões sistemáticas de ensaios de intervenções em assistência à saúde e a Best Evidence resume estudos individuais e revisões sistemáticas, conforme critérios explícitos para mérito científico e relevância clínica.

O MEDLINE é o maior banco mundial de dados gerais de literatura sobre a pesquisa biomédica, desde a pesquisa básica até a aplicada. Atualmente muitos bibliotecários e profissionais da área de saúde estão familizarizados com o MEDLINE, porque ele foi o primeiro banco de dados a disponibilizar a literatura para pesquisa eletrônica. Em virtude do seu enorme tamanho (cerca de 10 milhões de referências e contagens) e do amplo enfoque, ele possui uma ampla variedade de resultados sobre todas as pesquisas realizadas na área de saúde, mas é um desafio conseguir exatamente o que queremos. Hoje em dia existem outros bancos de dados especializados em pesquisa clínica que são menos conhecidos dos profissionais da saúde e bibliotecários, mas muito mais fáceis de serem usados e com maior probabilidade de fornecerem informações clinicamente úteis. A Cochrane Library e a Best Evidence são dois deles. Alguns "subgrupos” da MEDLINE para prática clínica são preparados por algumas companhias, particularmente para as especialidades maiores, como a cardiologia. Também podemos criar nosso próprio subgrupo clínico desenvolvendo uma estratégia de busca, que restrinja a busca no MEDLINE a revistas com maior probabilidade de fornecer artigos de relevância direta para a nossa prática. O MEDLINE deve ser utilizado pelo bibliotecário como apoio se não houver nenhum banco de dados na unidade de informação onde atua, ou se não for encontrada a informação procurada após a pesquisa em um banco de dados especializado. O MEDLINE é grátis a partir de muitas fontes, notavelmente a de sua criadora, a US National Library of Medicine (www.ncbi.nlm.nih.gov/PubMed). A BioMedNet também 
fornece acesso grátis ao MEDLINE (www.biomednet.com) com muitas melhorias, incluindo conexões com as fontes originais e referências relativas a cada entrada.

No Brasil, a biblioteca virtual de periódicos brasileiros SciELO (www.scielo.br) oferece acesso gratuito e integral para diversas revistas brasileiras.

Um complemento necessário para verificar bancos de dados e textos são os serviços de "alerta atualizado" que nos informam quando surgem novos estudos importantes relativos a tópicos de nosso interesse. Atualmente existem diversos recursos para nos mantermos atualizados, como por exemplo os descritos a seguir.

Começando com o ACP Journal Club em 1991, um número crescente de periódicos resume as melhores evidências das revistas tradicionais, fazendo a seleção conforme critérios explícitos de mérito, fornecendo resumos estruturados dos melhores estudos e comentários de especialistas para fornecer o contexto dos estudos e a aplicabilidade clínica dos seus achados. Essas novas revistas incluem Evidence-Basede Medicine, Evidence-Based Mental Health, Evidence-Based Nursing, Evidence-Based Health Care Policy and Practice e Evidence-Based Cardiovascular Medicine. Essas revistas fazem o que as revistas tradicionais gostariam de fazer, selecionando os melhores estudos. As revistas tradicionais não podem fazer isso porque podem publicar somente os artigos que os autores enviam. Assim, essas revistas de resumos fazem o que não conseguiríamos fazer por conta própria, isto é, resumem a melhor evidência a partir de estudos de alta qualidade selecionados das revistas de relevância para o interesse clínico dos profissionais da área da saúde, trazendo uma considerável contribuição para as pesquisas do bibliotecário médico.

A maioria dessas revistas de resumos com base em evidências são consultadas por generalistas. O bibliotecário médico pode indicar aos profissionais da área de saúde que construam seu próprio serviço de alerta atualizado. No momento, isso é fácil de obter através do índice das revistas relevantes de diferentes especialidades, enviado por serviços como Current Contentes, MEDLINE e Silverplatter. Além disso, estão surgindo na Web clubes de revistas, como, por exemplo, o Peds Journal Club 


\section{RELATO DE EXPERIÊNCIA}

(pedsccm.wustl.edu/Ebjournal_club.html), o Family Practice JC (POEMS) (www.infopoems.com/POEMS/POEMS_Home.htm),

Neurosurgery (http://www..brown.edu/Departments/Neurosurgery/EJC/journ.html) e o

Critical Care (http://ahsn.Ihs.on.ca). O SHARR fornece excelente conexão aos serviços com bases em evidências (www.shef.ac.uk/uni/academic/R-Z/scharr/ir/netting.html).

\section{CONSIDERAÇÕES FINAIS}

A atuação do bibliotecário médico expande-se na medida em que são utilizadas novas ferramentas de pesquisa para dar suporte aos profissionias da área da saúde. O seu exercício profissional em parceria com os profissionais da área médica amplia as possibilidades de encontrar os resultados esperados para a aplicação do uso da Medicina Baseada em Evidências, possibilitando para ambas as atividades profissionais direcionarem seus esforços na busca da melhor evidência científica. Enfim, a atuação do bibliotecário médico em instituições de saúde evoluirá na medida em que houver uma aproximação constante das necessidades do profissional da área da saúde.

\section{REFERÊNCIAS}

CAMPELLO, Bernadete Santos; CAMPOS, Carlita Maria. Fontes de informação especializada: características e utilização. 2. ed. Belo Horizonte: Ed. UFMG, 1993. 160 p.

DRUMMOND, José Paulo. et al. Medicina baseada em evidências. São Paulo: Atheneu, 1998.

GOMES, Marleide da Mota. Medicina baseada em evidências: princípios e práticas. Rio de Janeiro: Reichmann \& Affonso, 2001.

LANCASTER, F. W. Ameaça ou oportunidade? O futuro dos serviços de bibliotecas à luz das inovações tecnológicas. Tradução de: Gercina Ângela Borém Lima. Revista da Escola de Biblioteconomia da UFMG, Belo horizonte, v. 23 n. 1, p. 7-27, jan. / jun., 1994.

LIVELY, Lynn. Como gerenciar informações. Rio de Janeiro: Campus, 2002. 91 p. Medical Library Association (MLA). Disponível em: <http://www.mlahq.org>. Acesso em: 17 jan. 2004. 


\section{RELATO DE EXPERIÊNCIA}

POCOCK S. Clinical trials: a practical approach. Chinchester (UK): John Wiley \& Sons; 1983.

REGER, Nancy. Redefining Reference Services: Transitioning at One Public Library. Reference \& users services quarterly. v. 38, n. 1, p. 73-75, 1998.

\section{Fabiano Couto Corrêa da Silva}

Bibliotecário de Referência

Biblioteca Central - Universidade de Santa Cruz do Sul (UNISC)

Av. Independência, 2293 - 96815-900 - Santa Cruz do Sul - RS - Brasil Fone: (51) 84120042 e-mail:fabianos@unisc.br

Relato de Experiência aceito para publicação em: 06/ 2005 